\title{
Grazing incidence wide angle $x$-ray scattering at the wiggler beamline BW4 of HASYLAB
}

\author{
J. Perlich, ${ }^{1, a)}$ J. Rubeck, ${ }^{1}$ S. Botta, ${ }^{1}$ R. Gehrke, ${ }^{1}$ S. V. Roth, ${ }^{1}$ M. A. Ruderer, ${ }^{2}$ \\ S. M. Prams, ${ }^{2}$ M. Rawolle, ${ }^{2}$ Q. Zhong, ${ }^{2}$ V. Körstgens, ${ }^{2}$ and P. Müller-Buschbaum ${ }^{2}$ \\ ${ }^{1}$ HASYLAB, DESY, Notkestr. 85, 22607 Hamburg, Germany \\ ${ }^{2}$ Physik-Department, Technische Universität München, LS E13, James-Franck-Str. 1, 85748 Garching, \\ Germany
}

(Received 20 March 2010; accepted 20 August 2010; published online 15 October 2010)

\begin{abstract}
We present an upgrade of the available measurement techniques at the wiggler beamline BW4 of the Hamburger Synchrotronstrahlungslabor (HASYLAB) to grazing incidence wide angle x-ray scattering (GIWAXS). GIWAXS refers to an x-ray diffraction method, which, based on the measurement geometry, is perfectly suited for the investigation of the material crystallinity of surfaces and thin films. It is shown that the overall experimental GIWAXS setup employing a movable CCD-detector provides the capability of reliable and reproducible diffraction measurements in grazing incidence geometry. Furthermore, the potential usage of an additional detector enables the simultaneous or successive measurement of GIWAXS and grazing incidence small angle x-ray scattering (GISAXS). The new capability is illustrated by the microbeam GIWAXS measurement of a thin film of the conjugated polymer poly(3-octylthiophene) (P3OT). The investigation reveals the semicrystalline nature of the P3OT film by a clear identification of the wide angle scattering reflexes up to the third order in the [100]-direction as well as the first order in the [010]-direction. The corresponding microbeam GISAXS measurement on the present morphology complements the characterization yielding the complete sample information from subnanometer up to micrometer length scales. (C) 2010 American Institute of Physics. [doi:10.1063/1.3488459]
\end{abstract}

\section{INTRODUCTION}

The $\mathrm{x}$-ray wiggler beamline BW4 of DORIS III storage ring at Hamburger Synchrotronstrahlungslabor (HASYLAB) has been originally designed and equipped with instrumentation to measure ultrasmall angle $\mathrm{x}$-ray scattering (USAXS) in transmission scattering geometry with scattering angles on the order of $0.01^{\circ} .{ }^{1-3}$ In recent years, the beamline BW4 has become a dedicated materials science beamline, and the instrumentation has necessarily undergone significant refurbishments and upgrading. In addition to the standard USAXS geometry, small angle x-ray scattering (SAXS) setups were realized, thus increasing the accessible $q$-range. ${ }^{4,5}$

The regular operation of the x-ray scattering techniques in reflection (grazing incidence) scattering geometry such as grazing incidence small angle x-ray scattering (GISAXS) as well as grazing incidence ultrasmall angle $\mathrm{x}$-ray scattering (GIUSAXS) was established in recent years. ${ }^{6-8}$ Furthermore the equipment of the beamline with an ensemble of beryllium compound refractive lenses enables the optional focusing of the x-ray beam and reducing its size by one order of magnitude. ${ }^{4,9-11}$ The present availability of these four small angle scattering techniques (SAXS, USAXS, GISAXS, and GIUSAXS) as well as another recently applied novel technique of GISAXS microtomography provide the experimental basis for advanced characterization approaches at beam-

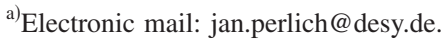

line BW4. ${ }^{12,13}$ The present upstream arrangement including the beamline optics and its technical specifications is described in detail elsewhere. ${ }^{2,4,11}$

However, the development of novel material systems in the application as thin films or isolated surface structures on top of a solid support has gained tremendous interest. ${ }^{14-22}$ For example, thin films for application in organic photovoltaics are formed by photoactive polymers and block copolymers as well as blends of photoactive polymers arranged in functional multilayered systems. ${ }^{23-25}$ Recently, we investigated the morphology of nanostructured titania thin films for hybrid photovoltaic devices including single titania thin films up to functional multilayer stacks present in real devices with GISAXS at beamline BW4. ${ }^{26-32}$ Besides, the morphological properties of these material systems, most of their possible applications are defined by physical characteristics of electrical, optical, or mechanical nature. By means of x-ray scattering, the morphology is commonly characterized with GI(U)SAXS at BW4 with accessible structure sizes $d$ in the typical range of $3 \mathrm{~nm} \leq d \leq 13000 \mathrm{~nm} \cdot{ }^{4,7}$ However, further characteristics such as the electrical material properties are mainly governed on atomic scale by the arrangement of atoms in a crystal lattice. Additionally, the specific crystal lattice might exhibit differences from the arrangement in bulk and in thin film geometry. In order to obtain crystalline structure sizes in thin films, x-ray scattering measurements at large angles are necessary due to the Bragg condition. ${ }^{33}$

Grazing incidence wide angle x-ray scattering (GI- 


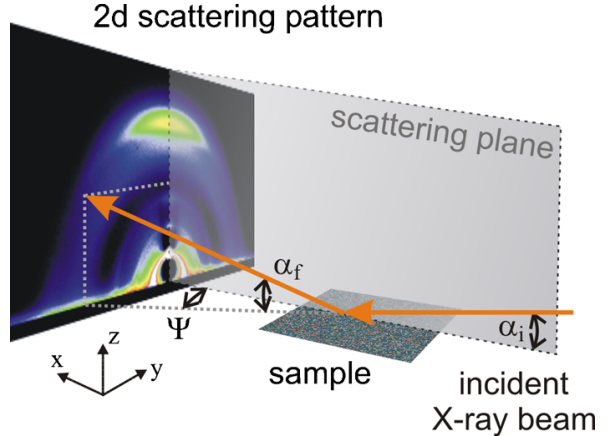

FIG. 1. (Color online) Schematic representation of the GIWAXS geometry. The sample surface is placed horizontally. The X-ray beam with wavelength $\lambda$ impinges on the sample surface with a fixed angle of incidence $\alpha_{i}$. The scattered beam is described by the exit angle $\alpha_{f}$ and the horizontal scattering angle $\Psi$. The area detector resembles the $q_{y}$ dependence along the horizontal axis and the $q_{z}$ dependence along the vertical axis (neglecting the small $q_{x}$ dependence). The detected two-dimensional scattering pattern shows the wide angle scattering originating from the sample. The primary beam and the intensity from the small angle scattering (GISAXS signal) are shielded with a rodlike beam stop.

WAXS) corresponds to an x-ray diffraction method, which is, because of the measurement geometry, perfectly adapted for the investigation of the crystallinity of surfaces and thin films. The GIWAXS measurement geometry is schematically shown in Fig. 1, where the diffuse scattering from the sample surface is collected with an area detector. A further denotation of the method, which rather meets the physical description as diffraction, is grazing incidence x-ray diffraction (GIXD). ${ }^{34-36}$ In GIXD, typically scans with high resolution are performed employing point or line detectors. However, in comparison to conventional x-ray powder diffraction (e.g., XRD) in the investigation of (ultra) thin films, the incident $\mathrm{X}$-ray beam interacts only with a very limited sample volume. Thus, similar to the methods of GISAXS and GIUSAXS in reflection geometry, the very small angle of incidence enables a sufficiently long penetration path inside the material and hence the collection of information from inside the thin film with high statistical significance.

In the present article, we report on GIWAXS as the most recent implementation of scattering techniques at the beamline BW4. The detailed description of the experimental setup is followed by an actual GIWAXS and GISAXS application example taken from the rapidly emerging field of conducting polymers. A summary and outlook section concludes the article.

\section{EXPERIMENTAL SETUP}

The GIWAXS experimental setup of the recent upgrade at BW4 is shown in Fig. 2. The accurate sample orientation to the incident $\mathrm{X}$-ray beam is provided by a combined sample stage including the translational movement in all three directions $(x, y, z)$ as well as sample rotations around those three axes. In comparison to the established conventional GISAXS setup, the MarCCD 165 area detector is placed in a reduced distance behind the sample (sample-to-detector distance $D_{\mathrm{SD}}$ ) enabling the sufficient recording of x-ray scattering at wide angles. Distances down to $0.1 \mathrm{~m}$ can be applied. The CCD is mounted on a combined translation stage for vertical

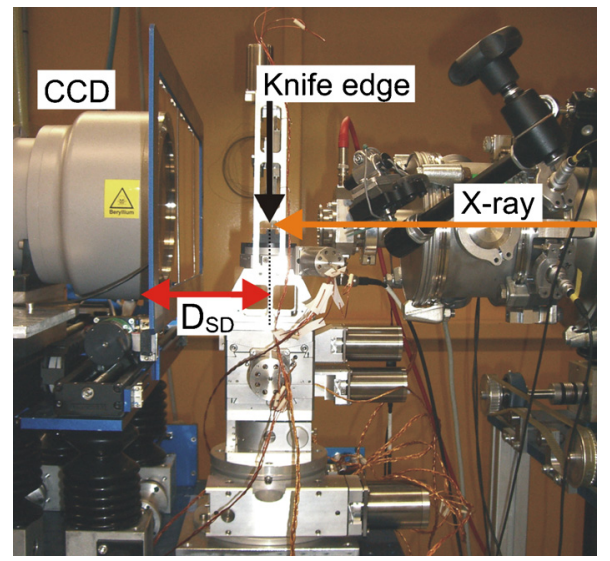

FIG. 2. (Color online) Photograph of the GIWAXS setup at BW4. The sample position is located where the arrows indicating the knife edge and the incoming X-ray beam coincide. CCD denotes the MarCCD 165 detector with the sample-to-detector distance $D_{\mathrm{SD}}$. The movable frame in front of the $\mathrm{CCD}$ accommodates the rodlike beam stop.

and horizontal movement of the detector, which enables a complete removal out of the beam pathway, required for the sample alignment, and the selective positioning of the scattering pattern on the detector. More flexibility is provided by the moveable beam stop frame in front of the detector, on which a rodlike beam stop with a typical diameter of $3 \mathrm{~mm}$ is fixed shielding the primary beam and the small angle $\mathrm{x}$-ray scattering signal (GISAXS signal) at the GIWAXS measurement.

A motor-controlled adjustable knife edge is mounted above the sample surface in order to reduce the diffuse scattering background and to remove the well-known scattering from Kapton. ${ }^{37,38}$ The Kapton foil is inevitably present as a robust window of the ionization chamber right in front of the sample, and the molecular aggregation in the aromatic polyimide film (Kapton) contributes with scattering in the small and wide angle regimes. ${ }^{38}$ The typical GIWAXS pattern for the measurement of a bare glass substrate in the absence of the knife edge is shown in Fig. 3(a). To distinguish the scattering originating from the Kapton from other scattering contributions, the measurement was performed at an slightly increased sample-to-detector distance $D_{\mathrm{SD}}=195 \mathrm{~mm}, \lambda$ $=0.1381 \mathrm{~nm}$, and $\alpha_{i}=0.2^{\circ}$ applying a moderately focused microbeam. In comparison, Fig. 3(b) shows the corresponding GIWAXS pattern with a closed knife edge approximately $300 \mu \mathrm{m}$ above the substrate surface. As result of applying the knife edge, the scattering background is considerably reduced, and the rather sharp Kapton ring at lower $q$ disappears completely. Hence, potential weak scattering from structures appearing at these scattering angles can be resolved. The remaining broader ringlike scattering at high $q$ can be clearly attributed to the scattering from the bare glass substrate. For WAXS experiments in transmission, where such a knife edge is not feasible anymore, a pin-hole aperture represented by a solid block of tungsten alloy (densimet) with a cylindrical hole of $1 \mathrm{~mm}$ in diameter is applied.

For the GIWAXS measurement, the sample has to be properly aligned in order to ensure an X-ray beam impinging onto the sample surface at the desired angle. For the align- 
(a)

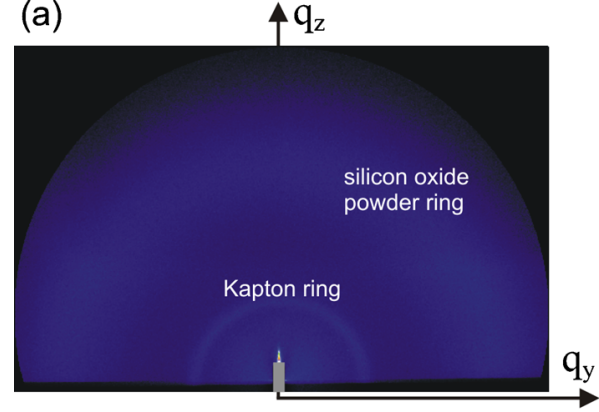

(b)

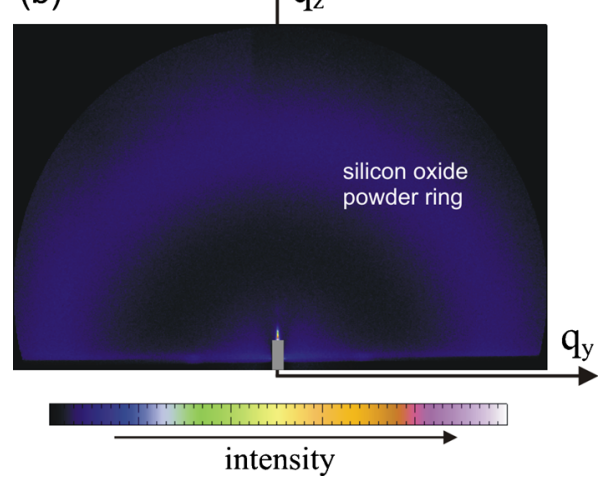

FIG. 3. (Color online) GIWAXS patterns of the bare glass substrate (a) without and (b) with the knife edge as recorded with the MarCCD 165 detector. For the two-dimensional intensity distribution, the horizontal (or out-of-plane) component of the scattering vector $q_{y}$ is plotted along the $x$-axis, and the vertical (or in-plane) component of the scattering vector $q_{z}$ is plotted along the $y$-axis. The intensity is shown on a linear scale. For color coding, see the scale bar (dark corresponds to low intensity and bright corresponds to high intensity). The high small angle scattering intensity (or GISAXS signal) is partially covered by a rodlike beam stop.

ment procedure, as depicted schematically in Fig. 4(a), the $\mathrm{CCD}$ and the beam stop frame are completely moved out of the beam pathway by the horizontal translation stage. This enables full access to the rear part of the beamline (behind the CCD), where a diode beam stop is situated originally employed as primary beam stop for the conventional GISAXS setup at BW4 [Fig. 4(b)]. ${ }^{4}$ In case the $\mathrm{x}$-ray beam is not obstructed by the sample, the full beam intensity is read out by the diode beam stop. During alignment, the exact vertical sample position (sample shading half of beam), as well as parallel sample orientation (tilt and inclination) with respect to the beam, is adjusted by recording the beam intensity during iteration of these parameters. After the alignment, the CCD is moved back to the desired position, and the beam stop frame is moved where the employed rodlike beam stop shields the primary beam and the GISAXS signal. Finally, the sample is moved to the selected angle of incidence and the GIWAXS measurement is performed. This configuration is shown schematically in Fig. 4(c). Taking all these steps, the accuracy available in GISAXS geometry is maintained together with the benefit of a fast sample alignment.

It is worthwhile to mention that the relevant wide angle scattering information in the present reflection setup is not hidden behind a beam stop, and therefore the resolution is only limited by the divergence of the primary beam, the sample-to-detector distance, and the spatial resolution of the detector. ${ }^{4}$ However, in the present setup only, the small angle

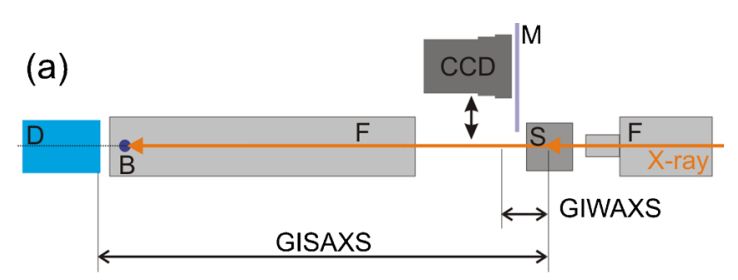

(b)

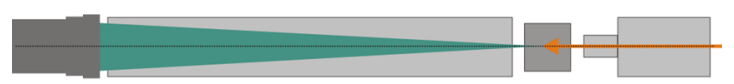

(c)

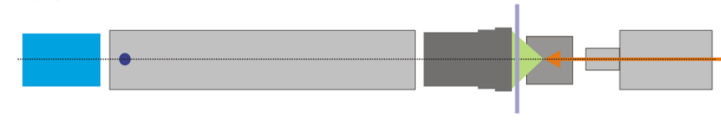

(d)

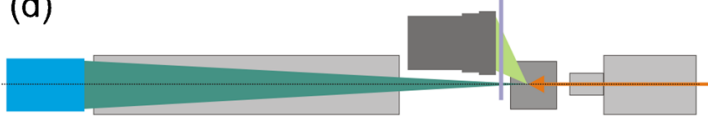

(e)

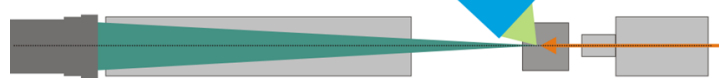

FIG. 4. (Color online) Top view of schematic representations of the different beamline configurations during sample alignment (a) and measurements for GISAXS (b), GIWAXS (c) and presently available configurations for successive or simultaneous GISAXS/GIWAXS (d) with exchanged detectors (e). The direction of the X-ray beam is indicated by the arrow. The basic components are presented by the moveable MarCCD detector (CCD) for GIWAXS with the moveable beam stop frame $(\mathrm{m})$, an additional area detector (d) for GISAXS, the sample (s), and the evacuated flight tubes (f) with a diode beam stop (b) mounted in the rear part. CCD (gray box) solely refers to the MarCCD detector, whereas the abbreviation D (blue box) denotes any additional detector, i.e., MarCCD 165, Pilatus $300 \mathrm{k}$ or $1 \mathrm{M}$.

scattering information, namely, the GISAXS signal, is hidden behind the rodlike beam stop resulting virtually in a resolution limit $q_{\min }$ defined by the diameter $d$ of the rodlike beam stop and $D_{\mathrm{SD}}$, e.g., for $d=3 \mathrm{~mm}$ and $D_{\mathrm{SD}}=125 \mathrm{~mm}$, we obtain $q_{\min }=0.72 \mathrm{~nm}^{-1}$. This limit is simply overcome by the complementary GISAXS measurement probing the GISAXS signal at typically larger sample-to-detector distances with high resolution in the absence of a rodlike beam stop.

\section{APPLICATION EXAMPLE}

GIWAXS is the technique of choice in order to investigate the degree of crystallinity in thin films and of structured surfaces. Commonly investigated material systems include conjugated polymers, block copolymers, lipids, and magnetic or semiconducting materials in a wide variety of applications. $^{39-46}$

To establish and prove the feasibility of GIWAXS at BW4, we applied this technique to a variety of different samples. Here we present the sample of a thin film of the conjugated and conducting polymer poly(3-octylthiophene) P3OT on top of a precleaned glass slide. P3OT belongs to the family of poly(3-alkylthiophene)s (P3ATs), which have a structure similar to hairy-rod polymers and form semicrys- 


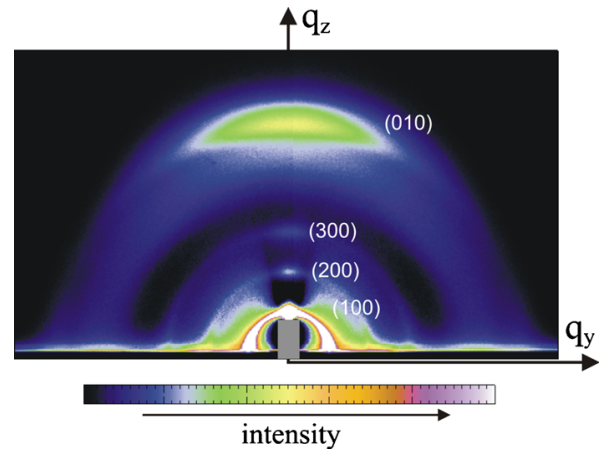

FIG. 5. (Color online) GIWAXS pattern of the thin conjugated polymer film of P3OT spin coated onto a glass slide as recorded with the MarCCD 165 detector. For the two-dimensional intensity distribution, the horizontal (or out-of-plane) component of the scattering vector $q_{y}$ is plotted along the $x$-axis, and the vertical (or in-plane) component of the scattering vector $q_{z}$ is plotted along the $y$-axis. The intensity is shown on a linear scale. For color coding, see the scale bar (dark corresponds to low intensity and bright corresponds to high intensity). The high small angle scattering intensity or GISAXS signal is covered by a rodlike beam stop (shaded area). The observed diffraction peaks are labeled according to the Miller indices $(h k l)$.

talline films with crystalline domains embedded in an amorphous matrix. ${ }^{40,47,48}$ P3ATs provide relatively high field effect mobilities and are therefore perfectly suited for applications such as organic light emitting diodes and organic field effect transistors as well as photovoltaic devices. $^{48,49}$

For the preparation of the thin film, P3OT $\left(M_{n}\right.$ $=34000 \mathrm{~g} / \mathrm{mol}$ ) was first dissolved in tetrahydrofuran yielding a solution with a concentration $c_{\mathrm{P} 3 \mathrm{OT}}=10 \mathrm{~g} / 1$. The thin film was spin coated out of the solution on a precleaned quartz glass slide and subsequently annealed in vacuum at a temperature $T=130{ }^{\circ} \mathrm{C}$ for $15 \mathrm{~min}$. The total film thickness was assigned to be $120 \mathrm{~nm}$ and confirmed with X-ray reflectometry (XRR) measurements. The sample was measured with the experimental GIWAXS setup shown in Fig. 2 with $D_{\mathrm{SD}}=125 \mathrm{~mm}$ at a wavelength $\lambda=0.1381 \mathrm{~nm}$ applying a moderately focused microbeam with a beam size at the sample position of $20 \times 40 \mu^{2}$ (vertical $\times$ horizontal). ${ }^{11}$ The microbeam impinged under an angle of incidence $\alpha_{i}$ $=0.2^{\circ}$ with respect to the sample surface resulting in a beam footprint of $5.7 \mathrm{~mm}$ in length. The recorded two-dimensional wide angle scattering pattern is presented in Fig. 5. The GIWAXS pattern is represented on linear scale (see Fig. 5) as a function of two perpendicular projections of the scattering vector $q$, namely, $q_{y}$ the out-of-plane (horizontal) scattering direction parallel to the sample surface and $q_{z}$ the vertical (in-plane scattering) direction. Due to the large density of crystallites, the GIWAXS pattern clearly indicates the presence of four peaks corresponding to the (100), (200), (300), and (010) diffraction peaks of the P3OT structure, which are strongest in the vertical direction. This indicates that the P3OT film has a well-organized structure with planar P3OT stacks oriented along an axis perpendicular to the substrate. In addition, the broader powderlike ring structure in the intensity distribution results primarily from the silicon oxide of the glass slide substrate, which has been confirmed by the GIWAXS measurements on a bare glass substrate as presented in Fig. 3.

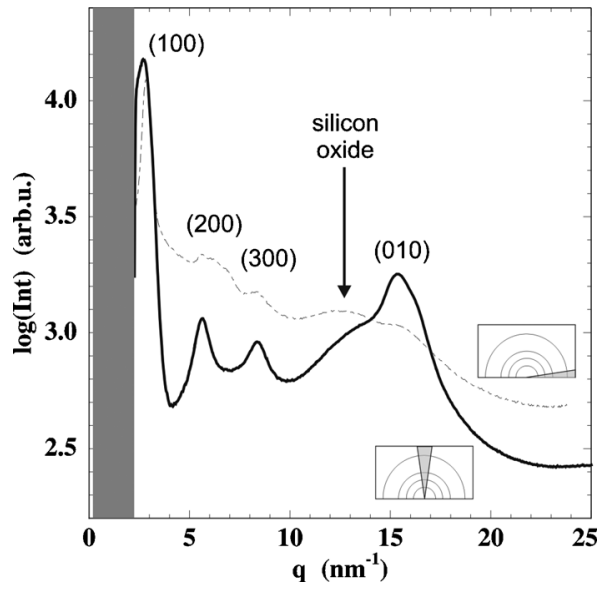

FIG. 6. One-dimensional scattering intensity curves $\log I(q)$ obtained by azimuthal integration of radial slices from the GIWAXS pattern as indicated by the pictograms: the solid line is for the azimuthal range of $0^{\circ}-5^{\circ}$ ( $q_{y}$-direction), and the dotted line is for the azimuthal range of $85^{\circ}-95^{\circ}$ ( $q_{z}$-direction). The $q$-position the powder ring originating from the glass substrate is indicated in the plot. The shaded area represents the beam stop where scattering data are unavailable.

For further analysis, Fig. 6 shows the one-dimensional scattering curves obtained by azimuthal integration of radial slices from the GIWAXS pattern. The qualitative comparison reveals that the scattering curve obtained by azimuthal integration in vertical direction is more pronounced than the scattering curve in out-of-plane direction. Therefore, the grazing incidence wide angle $\mathrm{x}$-ray scattering shows that the film is ordered vertically with respect to the substrate surface and highly anisotropic. The vertical ordering corresponds to the $\pi-\pi$ stacking between adjacent rodlike polymer backbones perpendicular to the substrate that self-organize into a lamellar supramolecular assembly or crystalline domains. ${ }^{40}$ The (100) diffraction peak and the corresponding higher order (200) and (300) diffraction peaks of the P3OT structure can be assigned to the lamellar repeating unit $a=2.3 \mathrm{~nm}$ resulting from the maxima position in $\log I(q)$ at $q_{(100)}$ $=2.7 \mathrm{~nm}^{-1}, q_{(200)}=5.6 \mathrm{~nm}^{-1}$, and $q_{(300)}=8.3 \mathrm{~nm}^{-1}$. The (010) diffraction peak at $q_{(010)}=15.3 \mathrm{~nm}^{-1}$ corresponds to a repeating unit $b=0.4 \mathrm{~nm}$ oriented perpendicular to $a$ and hence the distance between neighboring alkyl groups within the lamellar assembly (i.e., intraplanar spacing). ${ }^{50,51}$ The (010) diffraction peak is barely observed in the out-of-plane direction demonstrating a preferred orientation of the $\pi$-stack along the surface normal. Although the structures $a$ and $b$ are perpendicular to each other within each crystalline domain of the thin film, the orientation of different crystalline domains with respect to the sample surface has a certain angular distribution yielding the anisotropic GIWAXS scattering pattern.

The complementary microbeam GISAXS measurement of the P3OT thin film at beamline BW4 was performed at an increased sample-to-detector distance of $D_{\mathrm{SD}}=2162 \mathrm{~mm}$ but with the identical setup as presented for the GIWAXS measurement. The angle of incidence was slightly increased to $\alpha_{i}=0.386^{\circ}$, which is well above the critical angle of P3OT with $\alpha_{c, \mathrm{P} 3 \mathrm{OT}}=0.14^{\circ}$ and of quartz glass $\alpha_{c, \mathrm{SiO}_{2}}=0.19^{\circ}$. The GISAXS pattern is presented on logarithmic scale in Fig. 7. 


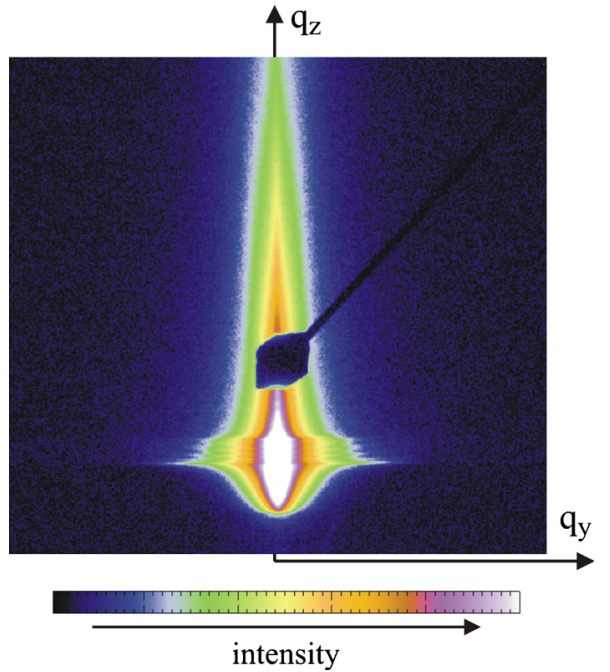

FIG. 7. (Color online) The corresponding GISAXS pattern of the thin conjugated polymer film of P3OT spin coated onto a glass slide as recorded with the MarCCD 165 detector. For the two-dimensional intensity distribution, the horizontal (or out-of-plane) component of the scattering vector $q_{y}$ is plotted along the $x$-axis, and the vertical (or in-plane) component of the scattering vector $q_{z}$ is plotted along the $y$-axis. The intensity is shown on a logarithmic scale. For color coding, see the scale bar (dark corresponds to low intensity and bright corresponds to high intensity). The detected 2D scattering pattern shows the diffuse scattering with a Yoneda region (offspecular scattering maxima) and the specular peak (shielded by a small pointlike beam stop that protects the detector from the very high intensity of the specularly reflected beam).

The diffuse scattering signal exhibits a rather narrow intensity distribution along the scattering plane (see Fig. 1). At the Yoneda region $\alpha_{f} \approx \alpha_{c, \mathrm{P} 3 \mathrm{OT}, \mathrm{SiO}_{2}}$, the intensity is broadened and extends toward higher $q_{y} .{ }^{7}$ Hence, the scattering pattern indicates the presence of a smooth P3OT thin film with low surface and interfacial roughness as well as a low degree of lateral order in the thin film. Furthermore, the ripplestructure or intensity modulation at the Yoneda region indicates interference effects originating from correlated roughness of thin film and substrate. ${ }^{52-54}$ For the further quantitative analysis, line cuts in the vertical and horizontal direction from the GISAXS pattern are displayed in Fig. $8 .{ }^{55}$ The vertical or detector cut along the scattering plane, representing structural information perpendicular to the sample surface, is shown in Fig. 8(a). The zoom-in of the Yoneda region (inset) clearly shows a pronounced modulation in the scattering intensity for $\alpha_{f}<\alpha_{c, \mathrm{SiO}_{2}}$ originating from correlated roughness. ${ }^{53}$ In more detail, the average distance of the observed maxima can be calculated to $\Delta q_{z}=0.01146 \mathrm{~nm}^{-1}$, corresponding to a characteristic length scale normal to the sample surface of $548 \mathrm{~nm}$. Because the obtained length exceeds the P3OT film thickness $(120 \mathrm{~nm})$ considerably, the observed modulation effect is caused by the roughness correlated thin film in a dynamical waveguide manner. ${ }^{54}$ The corresponding horizontal cut shown in Fig. 8(b) was performed at the critical angle $\alpha_{c \text {,РзОт }}$ and contains information about lateral structures of the P3OT thin film. For analysis, the characteristic lateral length scales were modeled with form factors. A Lorentzian size distribution of the characteristic lateral length scale was assumed to account for statistical deviations. In the fit (solid line), the experimentally de- (a)

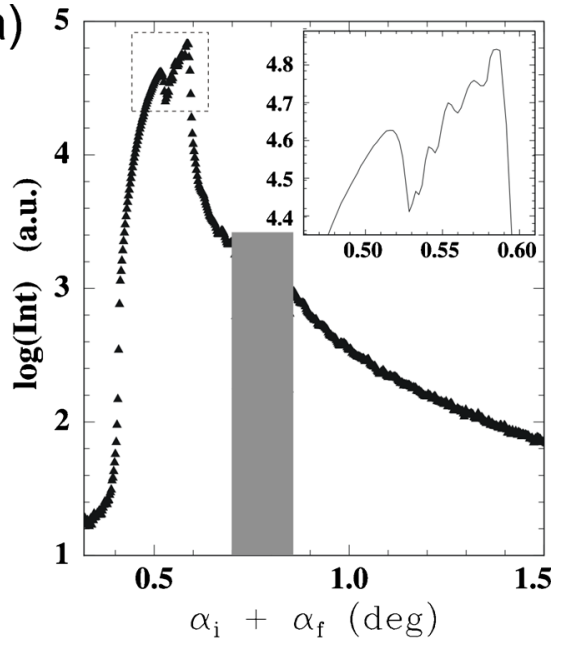

(b)

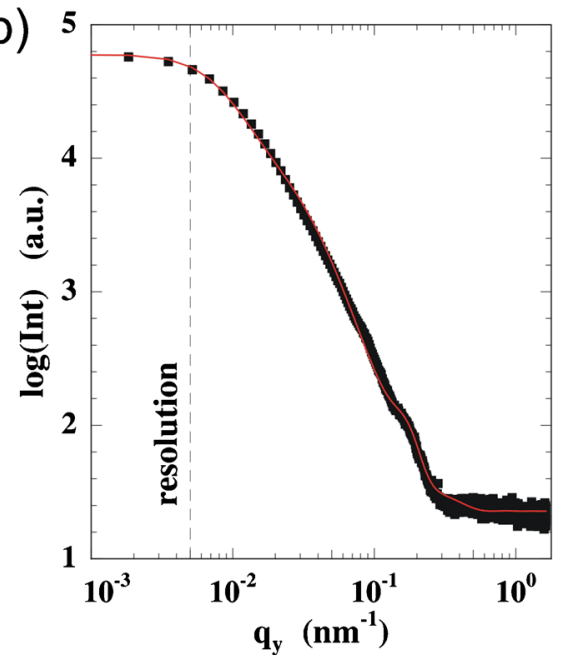

FIG. 8. (Color online) (a) Vertical cut of the GISAXS pattern at horizontal scattering angle $\Psi=0^{\circ}\left(q_{y}=0\right)$. The scattering intensity is plotted vs the sum of $\alpha_{i}$ and $\alpha_{f}$. The shaded area corresponds to the position of the specular beam stop. The inset shows a zoom-in of the Yoneda region. The visible intensity modulation corresponds to correlated roughness of the P3OT film on top of the glass substrate. (b) Double-logarithmic plot of the horizontal cut (symbols) of the GISAXS pattern as a function of the $q_{y}$ component of the scattering vector. The solid line is the mathematical fit for determining the characteristic lateral length scales of the P3OT thin film. Features originating from the obtained characteristic lateral length scales are marked with the arrows. The dashed line indicates the resolution limit of the GISAXS experiment.

termined resolution function was taken into account. ${ }^{7}$ Two characteristic lateral length scales of $\Lambda_{A}=40 \mathrm{~nm}$ and $\Lambda_{B}$ $=800 \mathrm{~nm}$ were obtained. Because the thin film consists of pure P3OT homopolymer with a homogeneous thickness $(120 \mathrm{~nm})$ as verified with XRR, for this closed homopolymer thin film, the obtained characteristic lateral length scales originate solely from its inner structure. This inner structure comprises domains of crystallites and the obtained characteristic lateral length scales $\Lambda_{A, B}$ can be attributed to average domain sizes. Furthermore, the absence of any pronounced peak in the horizontal cut corresponds to a rather polydisperse size distribution of these domains.

\section{SUMMARY AND OUTLOOK}

The recent upgrade of x-ray scattering techniques at the beamline BW4 results in the availability of GIWAXS for the 
measurement of dedicated samples in reflection scattering geometry. The successful establishment and feasibility of GIWAXS at BW4 are illustrated with the example of the measurement of a thin film of P3OT by the newly established technique. With the presented GIWAXS setup, employing the MarCCD 165 area detector in a symmetric position around the primary beam a $q$-range up to $25 \mathrm{~nm}^{-1}$ (corresponding to $0.25 \mathrm{~nm}$ in real space) is accessible with a resolution of $\Delta q=0.03 \mathrm{~nm}^{-1}$. Since the MarCCD detector is moveable in vertical and horizontal directions, an extension of the accessible $q$-range is also possible. Given the maximum offset of the detector perpendicular to the x-ray beam, the smallest probable length decreases to $0.12 \mathrm{~nm}$. Furthermore, the usage of an additional detector, i.e., MarCCD 165, Pilatus $300 \mathrm{k}$ or $1 \mathrm{M}$ single-photon count detectors, enables in situ experiments where both the small (GISAXS) and wide (GIWAXS) angle scattering intensities are recorded successively or simultaneously. One beamline configuration for the simultaneous measurement is presented schematically in Fig. 4(d). Moreover, the exchange of the detectors combined with the "lightweight" construction of the $300 \mathrm{k}$ single-photon count detectors enables a rather freely chosen positioning of the detector around the sample [see Fig. 4(e)]. Thus, despite limitation imposed by the detector covers, basically any selected $q$-range will become accessible for measurements in GIWAXS mode. The application of these two options depends solely on the selected GIWAXS mode, whether operated in symmetric or asymmetric position of the detector around the primary beam. Nevertheless, the once aligned sample can be completely characterized with GISAXS and GIWAXS under identical measurement conditions resulting in the illumination of exactly the same sample area. Combined with the usage of the moderately focused microbeam, this experimental setup represents a powerful tool for obtaining the locally resolved morphology and corresponding crystallinity of gradients in thin films.

In summary, the recent upgrade makes the beamline BW4 a most versatile beamline with a unique range of available sample-to-detector distances from $0.1 \mathrm{~m} \leq D_{\mathrm{SD}}$ $\leq 13.5 \mathrm{~m}$ accessing $0.0005 \mathrm{~nm}^{-1} \leq q \leq 25 \mathrm{~nm}^{-1}$ in reciprocal space.

\section{ACKNOWLEDGMENTS}

M.A.R. acknowledges the Bavarian State Ministry of Science, Research and Arts for funding through the International Graduate School "Materials Science of Complex Interfaces" (CompInt). This work was financially supported by DFG in the priority program for nano- and microfluids SPP1164 (Grant No. MU1487/2).

${ }^{1}$ R. Gehrke, Rev. Sci. Instrum. 63, 455 (1992).

${ }^{2}$ R. Gehrke, M. Bark, D. Lewin, and S. Cunis, Rev. Sci. Instrum. 66, 1354 (1995).

${ }^{3}$ A. Endres, U. Lode, G. v. Krosigk, M. Bark, S. Cunis, R. Gehrke, and W. Wilke, Rev. Sci. Instrum. 68, 4009 (1997).

${ }^{4}$ S. V. Roth, R. Döhrmann, M. Dommach, M. Kuhlmann, I. Kröger, R. Gehrke, H. Walter, C. Schroer, B. Lengeler, and P. Müller-Buschbaum, Rev. Sci. Instrum. 77, 085106 (2006).

${ }^{5}$ S. Förster, A. Timmann, C. Schellbach, A. Frömsdorf, A. Kornowski, H. Weller, S. V. Roth, and P. Lindner, Nature Mater. 6, 888 (2007).
${ }^{6}$ P. Müller-Buschbaum, P. Vanhoorne, V. Scheumann, and M. Stamm, Europhys. Lett. 40, 655 (1997).

${ }^{7}$ P. Müller-Buschbaum, Anal. Bioanal. Chem. 376, 3 (2003).

${ }^{8}$ P. Müller-Buschbaum, Prog. Colloid Polym. Sci. 132, 23 (2006).

${ }^{9}$ B. Lengeler, C. Schroer, M. Kuhlmann, B. Benner, T. F. Günzler, O. Kurapova, F. Zontone, A. Snigirev, and I. Snigireva, J. Phys. D 38, A218 (2005).

${ }^{10}$ A. Timmann, S. V. Roth, S. Fischer, and S. Förster, Appl. Phys. Lett. 91, 213102 (2007).

${ }^{11}$ A. Timmann, R. Döhrmann, T. Schubert, H. Schulte-Schrepping, U. Hahn, M. Kuhlmann, R. Gehrke, S. V. Roth, A. Schropp, C. Schroer, and B. Lengeler, Rev. Sci. Instrum. 80, 046103 (2009).

${ }^{12}$ C. G. Schroer, M. Kuhlmann, S. V. Roth, R. Gehrke, N. Stribeck, A. Almendarez-Camarillo, and B. Lengeler, Appl. Phys. Lett. 88, 164102 (2006).

${ }^{13}$ M. Kuhlmann, J. M. Feldkamp, J. Patommel, S. V. Roth, A. Timmann, R. Gehrke, P. Müller-Buschbaum, and C. G. Schroer, Langmuir 25, 7241 (2009).

${ }^{14}$ J. S. Gutmann, P. Müller-Buschbaum, and M. Stamm, Appl. Phys. A: Mater. Sci. Process. 74, s463 (2002).

${ }^{15}$ S. V. Roth, H. Walter, M. Burghammer, C. Riekel, B. Lengeler, C. Schroer, M. Kuhlmann, T. Walther, R. Domnick, and P. MüllerBuschbaum, Appl. Phys. Lett. 88, 021910 (2006).

${ }^{16}$ A. Frömsdorf, A. Kornowski, S. Pütter, H. Stillrich, H. P. Oepen, and L.-T. Lee, Small 3, 880 (2007).

${ }^{17}$ D. R. Rueda, A. Nogales, J. J. Hernandez, M. C. Garcia-Gutierrez, T. A. Ezquerra, S. V. Roth, M. G. Zolotukhin, and R. Serna, Langmuir 23, 12677 (2007).

${ }^{18}$ H. Yokoyama, C. Dutriez, L. Li, T. Nemoto, K. Sugiyama, S. Sono, H. Masunaga, M. Takata, and H. Okuda, J. Chem. Phys. 127, 014904 (2007).

${ }^{19}$ M. M. Abul Kashem, J. Perlich, A. Diethert, W. Wang, M. Memesa, J. S. Gutmann, E. Majkova, I. Capek, S. V. Roth, W. Petry, and P. MüllerBuschbaum, Macromolecules 42, 6202 (2009).

${ }^{20}$ E. Metwalli, J.-F. Moulin, J. Perlich, W. Wang, A. Dietert, S. V. Roth, and P. Müller-Buschbaum, Langmuir 25, 11815 (2009).

${ }^{21}$ H. Okuda, M. Kato, S. Ochiai, and Y. Kitajima, Appl. Phys. Express 2, 126501 (2009).

${ }^{22}$ W. Wang, G. Kaune, J. Perlich, C. M. Papadakis, A. M. B. Koumba, A. Laschewsky, K. Schlage, R. Röhlsberger, S. V. Roth, R. Cubitt, and P. Müller-Buschbaum, Macromolecules 43, 2444 (2010).

${ }^{23}$ M. Al-Hussein, M. A. Ruderer, E. Metwalli, V. Körstgens, U. Vainio, S. V. Roth, R. Döhrmann, R. Gehrke, R. Gebhardt, M. Burghammer, and P. Müller-Buschbaum, Macromolecules 42, 4230 (2009).

${ }^{24}$ M. A. Ruderer, E. Metwalli, W. Wang, G. Kaune, S. V. Roth, and P. Müller-Buschbaum, ChemPhysChem 10, 664 (2009).

${ }^{25}$ M. A. Ruderer, V. Körstgens, E. Metwalli, M. Al-Hussein, U. Vainio, S. V. Roth, R. Döhrmann, R. Gehrke, R. Gebhardt, M. Burghammer, and P. Müller-Buschbaum, Nucl. Instrum. Methods Phys. Res. B 268, 403 (2010).

${ }^{26}$ P. Müller-Buschbaum, J. Perlich, M. M. A. Kashem, L. Schulz, S. V. Roth, Y.-J. Cheng, and J. S. Gutmann, Phys. Status Solidi (RRL) 1, 119 (2007).

${ }^{27}$ G. Kaune, W. Wang, E. Metwalli, M. Ruderer, R. Roßner, S. V. Roth, and P. Müller-Buschbaum, Eur. Phys. J. E 26, 73 (2008).

${ }^{28}$ J. Perlich, M. Memesa, A. Diethert, E. Metwalli, W. Wang, S. Roth, A. Timmann, J. S. Gutmann, and P. Müller-Buschbaum, Phys. Status Solidi (RRL) 3, 118 (2009).

${ }^{29}$ J. Perlich, M. Memesa, A. Diethert, E. Metwalli, W. Wang, S. Roth, A. Timmann, J. S. Gutmann, and P. Müller-Buschbaum, ChemPhysChem 10, 799 (2009).

${ }^{30}$ J. Perlich, G. Kaune, M. Memesa, J. S. Gutmann, and P. MüllerBuschbaum, Philos. Trans. R. Soc. London, Ser. A 367, 1783 (2009).

${ }^{31}$ G. Kaune, M. Memesa, R. Meier, M. A. Ruderer, A. Diethert, S. V. Roth, M. D'Acunzi, J. S. Gutmann, and P. Müller-Buschbaum, ACS Appl. Mater. Interfaces 1, 2862 (2009).

${ }^{32}$ M. Memesa, S. Weber, S. Lenz, J. Perlich, R. Berger, P. MüllerBuschbaum, and J. S. Gutmann, Energy Environ. Sci. 2, 783 (2009).

${ }^{33}$ W. L. Bragg, Proc. Cambridge Philos. Soc. 17, 43 (1913).

${ }^{34}$ R. Feidenhans'1, Surf. Sci. Rep. 10, 105 (1989).

${ }^{35} \mathrm{H}$. Dosch, Critical Phenomena at Surfaces and Interfaces: Evanescent $X$-Ray and Neutron Scattering (Springer, New York, 1992).

${ }^{36}$ J. Als-Nielsen and D. McMorrow, Elements of Modern X-Ray Physics (Wiley, New York, 2001).

${ }^{37}$ G. Conte, L. D'Ilario, N. V. Pavel, and E. Giglio, J. Polym. Sci., Polym. Phys. Ed. 14, 1553 (1976). 
${ }^{38}$ B. J. Kim, Y. Miyamoto, B. Ma, and J. M. J. Fréchet, Adv. Funct. Mater. 19, 2273 (2009).

${ }^{39}$ S. Isoda, H. Shimada, M. Kochi, and H. Kambe, J. Polym. Sci., Polym. Phys. Ed. 19, 1293 (1981).

${ }^{40}$ H. G. O. Sandberg, G. L. Frey, M. N. Shkunov, H. Sirringhaus, R. H. Friend, M. M. Nielsen, and C. Kumpf, Langmuir 18, 10176 (2002).

${ }^{41}$ J. W. Andreasen, M. Jørgensen, and F. C. Krebs, Macromolecules 40, 7758 (2007).

${ }^{42}$ G. Rodríguez, L. Barbosa-Barros, L. Rubio, M. Cócera, A. Díez, J. Estelrich, R. Pons, J. Caelles, A. D. la Maza, and O. López, Langmuir 25, 10595 (2009).

${ }^{43}$ H. P. Jakobsen, J. W. Andreasen, K. Norrman, and F. C. Krebs, Thin Solid Films 516, 2203 (2008).

${ }^{44}$ T. Hirai, M. Leolukman, S. Jin, R. Goseki, Y. Ishida, M. Kakimoto, T. Hayakawa, M. Ree, and P. Gopalan, Macromolecules 42, 8835 (2009).

${ }^{45}$ A. Turković, Z. Crnjak-Orel, P. Dubček, and H. Amenitsch, Sol. Energy Mater. Sol. Cells 59, 387 (1999).

${ }^{46}$ L. Favre, V. Dupuis, E. Bernstein, P. Mélion, A. Peréz, S. Stanescu, T.
Epicier, J.-P. Simon, D. Babonneau, J.-M. Tonnerre, and J.-L. Hodeau, Phys. Rev. B 74, 014439 (2006).

${ }^{47}$ Y. D. Park, D. H. Kim, Y. Jang, J. H. Cho, M. Hwang, H. S. Lee, J. A. Lim, and K. Cho, Org. Electron. 7, 514 (2006).

${ }^{48}$ M. C. Gurau, D. M. Delongchamp, B. M. Vogel, E. K. Lin, D. A. Fischer, S. Sambasivan, and L. J. Richter, Langmuir 23, 834 (2007).

${ }^{49}$ X. Xiao, Z. Hu, Z. Wang, and T. He, J. Phys. Chem. B 113, 14604 (2009).

${ }^{50}$ X. Qiao, X. Wang, and Z. Mo, Synth. Met. 118, 89 (2001).

${ }^{51}$ A. Urbina, E. Palacios-Lidón, C. Miguel, B. Pérez-García, R. GarcíaValverde, J. Abellán, and J. Colchero, Eur. Phys. J.: Appl. Phys. 37, 283 (2007).

${ }^{52}$ V. Holý and T. Baumbach, Phys. Rev. B 49, 10668 (1994).

${ }^{53}$ P. Müller-Buschbaum, J. S. Gutmann, C. Lorenz, T. Schmitt, and M. Stamm, Macromolecules 31, 9265 (1998).

${ }^{54}$ M. M. A. Kashem, J. Perlich, L. Schulz, S. V. Roth, and P. MüllerBuschbaum, Macromolecules 41, 2186 (2008).

${ }^{55}$ P. Müller-Buschbaum, S. V. Roth, M. Burghammer, A. Diethert, P. Panagiotou, and C. Riekel, Europhys. Lett. 61, 639 (2003). 